\begin{tabular}{c|c|c|}
\hline \hline & International Journal of Current Research in & \\
\hline & Biosciences and Plant Biology \\
\hline \\
\hline \hline
\end{tabular}

\title{
Ethnomedicinal Knowledge of Galo Tribe from Arunachal Pradesh, India
}

\author{
Pallabi Bharali, Binay Singh and Chaman Lal Sharma* \\ Department of Forestry, North Eastern Regional Institute of Science and Technology (Deemed University), Nirjuli-791109, \\ Arunachal Pradesh, India. \\ *Corresponding author.
}

\begin{abstract}
Use of plant resources for fulfillment of various requirements of any community may be regarded as a part of a culture's traditional knowledge. The tribes of Arunachal Pradesh are found to be rich in traditional knowledge system and are using their indigenous methods in treatments of different diseases. The present paper deals with the detail study of medicinal plants used by Galo tribe of West Siang district, Arunachal Pradesh, using a quantitative consensus analysis. A total of 45 species belonging to 32 families used for medicinal and general health purposes were identified and included with relevant information. An informant consensus $\left(\mathrm{F}_{\mathrm{IC}}\right)$ analysis revealed a high level of homogeneity among the informant's knowledge on various ethnomedicinal plants. A high consensus factor was observed for dermatological disorder (0.82) and low in fever (0.5). Among different plant parts, leaves were used in most of the cases for treatment of various diseases by the Galo tribe.
\end{abstract}

\section{Introduction}

Arunachal Pradesh is the largest states of North East India with a geographic area of $83,743 \mathrm{sq} \mathrm{km}$ and has a large number of tribal groups scattered within the state. The Galo tribe with maximum population is concentrated in West Siang District of Arunachal Pradesh (Dagyom and Gopi, 2006). They have tremendous traditional knowledge on the utilization of natural resources related to conservation of food and medicinal plants (Bora et al., 2012). The indigenous knowledge is mostly transmitted from one to next generation orally. The tribal people mostly collect the wild plants from the forest adjacent to their villages and cultivate most preferred species in their home gardens.

Due to rich heritage and cultural diversity, the state is an important region for ethnobotanical studies. Some important documented publications on ethnobotanical knowledge of various communities reveal a large numbers of wild plants used in various ways. A number of information is available to understand the ethnomedico-botany of the indigenous communities (Kohli,2001; Tag and Das, 2004; Mutem and Das, 2005; Kala, 2005; Dutta and Bhattacharjya, 2005; Tarak et al., 2009; Goswami et al., 2009; Sen et al., 2009; Srivastava and Adi Community, 2009; Srivastava and Nyshi Community, 2010; Doley et al., 2010; Jeri et al., 2011; Namsa et al ,2011; Khongsai et al.,2011; Nimachow et al., 2011 and 2012; Bora et al., 2012; Das et al., 2013). However, there is no report on the ethnomedicinal plants used by the Galo tribe of west Siang district so far. Therefore, the present study is undertaken to document the ethno medicinal use pattern and analyse the informant consensus factor $\left(\mathrm{F}_{\mathrm{IC}}\right)$ to estimate use variability of medicinal plants used by the Galo tribe of Arunachal Pradesh. 


\section{Materials and methods}

\section{Study area}

West Siang district is located in central position of Arunachal Pradesh between $93^{0} 57^{\prime} \mathrm{E}$ longitude and $27^{0} 20^{\prime} \mathrm{N}$ latitudes. The district is surrounded by the Tibet region of China in the North, East Siang district in the east, Upper Subansiri and Lower Subansiri Districts in the west. Its southern boundary adjoins with Dhemaji district of Assam and lies in the foot hill range with hills of low altitudes (Fig. 1). The study area covers Aalo Circles in West Siang district of Arunachal Pradesh and a total of 10villages namely Pakam, Zirdin, Raglam, Darka, Eyi, Paya, Gune, Bene, Pobdi, Kabu and Kamdi inhabited by Galo tribe were selected for field survey. Total population of West Siang district is112,274 persons (2011census). The average minimum and maximum temperature of the area is $10.21^{\circ} \mathrm{C}$ in winter and $38^{\circ} \mathrm{C}$ in summer respectively and the annual rainfall is about $2061.10 \mathrm{~mm}$.

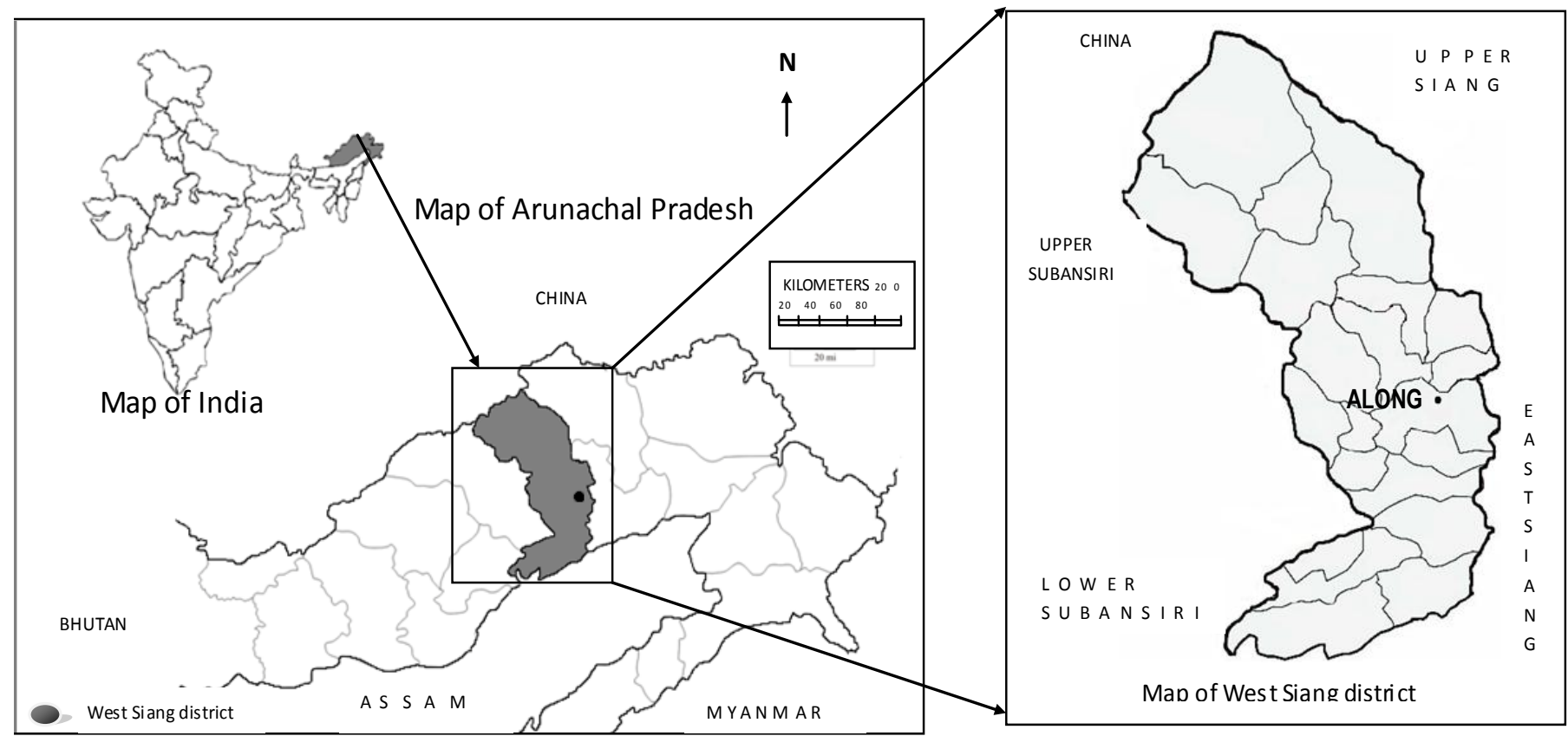

Fig. 1: Location of the study area.

\section{Ethnographic profile of Galo}

The Galo speak a Tani language Gallong which is a Sino-Tibetan language of the Tani group and are descendant of the Abo Tani (Post, 2007). In the past it is known as Duba, Doba, Dobah Abor, Gallong Abor, Galong, Galong Adi, etc. The tribe is composed of several groups inhabiting a compact area and comprise of many villages that are culturally and socially linked together(Bora et al.,2012). Galos mainly depend on the cultivation, practice shifting cultivation because of their remoteness. Donyi-Polo religious tradition persists to a degree in most Galo, although Christianity is on the rise in recent years especially in the foothill areas (Post, 2007). Mopin is the main festival of the tribe is celebrated in the month of April for social prosperity and wealth. Galos have tremendous traditional knowledge on use natural resources to a great extent.

\section{Data collection}

Secondary information was collected from various sources like office booklets, statistical abstracts, books and journals before field study to collect data on the locality, people etc. Detailed Ethnobotanical surveys were conducted during the year from 2011 to 2013 to collect the data. Over 120 informants were interviewed of which 80 key informants were chosen equally distributed among different age groups. Informants were selected by making discussion with the Head of the respective village to document the plants. Relevant information was collected on the basis of frequent interviews with the selected experienced old people and the same has been noted down in household schedules and field diary. Voucher specimens for all the ethno medicinally important plants were collected to ensure proper identification of the plant. 
Specimens were identified with the help of herbarium materials, experts and by consulting standard floras (Choudhery, 1996 and Hooker, 1894). All the specimens were deposited in the herbarium of Forestry Department of North Eastern Regional Institute of Science and Technology (NERIST), Nirjuli, Arunachal Pradesh.

To evaluate the variability of the use of medicinal plants and to determine homogeneity on the informant's knowledge, the informants consensus factor $\left(\mathrm{F}_{\mathrm{IC}}\right)$ was calculated (Heinrich et al., 1998) given as below:

$$
\mathrm{F}_{\mathrm{IC}}=\mathrm{N}_{\mathrm{ur}}-\mathrm{N}_{\mathrm{t}} /\left(\mathrm{N}_{\mathrm{ur}}-1\right)
$$

This factor ranges between 0 and 1 , where a high value means a good indicator for high rate of informant consensus. $\mathrm{N}_{\mathrm{ur}}$ is the number of use reports by informants for usage of particular illness and $\mathrm{N}_{\mathrm{t}}$ refers to number of species used for particular illness category by all informants. The majority of ailment types are grouped into predefined ethnobotanical categories (Heinrich, 2000; Ragupathy et al., 2008; Arwa et al., 2010; Njoroge and Bussmann, 2006; Tolossa et al., 2013), with the additions of a few other ailment categories, which were commonly mentioned during the interviews as they were prevalent among the communities of the selected site. The use of "General metabolism categories" is adopted here as recommended by other ethnobotanical researchers (Heinrich, 2000; Ragupathy et al., 2008). In each ailment category parameter such as number of species, genera and families used and plant parts used has been presented. Overall 47 illnesses reported were grouped into 8 major categories.

\section{Results and discussion}

The study reveals the use of various plant species for curing different ailments among Galo tribe of West Siang District, Arunachal Pradesh. The plants used for preparation of traditional medicine are mostly collected from the wilderness. However, some expert practitioners have their own herbal gardens that supply the useful raw materials. Majority of the plants were used more or less for the same purpose with only slight variations in recipes informed by most of the informants. All together 45 plant species belonging to 32 families and 41 genera have been documented in this study (Table 1).

The highest numbers of plants were from family Asteraceae followed by Musaceae, Piperaceae, Rutaceae, Solanaceae, Clusiaceae, Araceae families (Fig. 2). Among all the plant parts used ethnomedicinally, leaves are used mostly used (38\%), followed by fruits (26\%), seeds and stem (7\%) (Fig. 3).

As per habits, herbs (38\%) were the most used ethnomedicinal species followed by the trees $(27 \%)$, shrubs (24\%) and climbers (11\%) (Fig. 4), the use of herbal formulation in internaluse was much higher $(75 \%)$ than that used externally (25\%). Medicinally oral application is dominant mode of use when the crude drug type was recorded the decoction and juice extract was found more common than eaten raw. About $82 \%$ plants grown in wild and only $18 \%$ are cultivated in their garden.

The informant consensus analysis was carried out to test the reliability of ethnobotanical data as revealed by the practitioners (Table 2). In the study, the informant consensus of plant usage as ethnomedicine among the Galos resulted in $\mathrm{F}_{\mathrm{IC}}$ factors ranging from 0.5 to 0.82 per illness category. As per literature survey, high informant consensus $\left(\mathrm{F}_{\mathrm{IC}} 0.56\right.$ and $\mathrm{F}_{\mathrm{IC}}$ 0.73) was recorded among Monpa ethnic group for dermatological disorder and for general health among Adi tribe (Nimasow et al., 2013 and 2014) of Arunachal Pradesh respectively.

In the present study highest $\mathrm{F}_{\mathrm{IC}}$ was found in the dermatological disorder (0.82) followed by general health (0.79), gastrointestinal disorder (0.75) and Pain (0.71), 65 numbers of citations were recorded for dermatological disorder with use of 12 species. Similarly, for general health with 55 citations with 12 species in use and for gastrointestinal disorder 25 species are reported with 97 citations. Other category included diseases such as Respiratory system disorder, genitourinary system disorder, fever, jaundice for which the $\mathrm{F}_{\mathrm{IC}}$ value was low ranging from 0.5 to 0.66 . The high $F_{I C}$ factor indicates the homogeneity of informants. The more homogeneity for the dermatological disorder, general health, gastrointestinal disorder and pain may be due to availability and easily accessible of plants used in these diseases or the quick effect of these plants on diseases is the another case of more homogeneity.

A low consensus factor indicates the higher number of plant species used in this category but lesser homogeneity among informants. The low consensus factor $\left(F_{I C} 0.50\right)$ for fever category may be due to availability of easily accessible pharmaceuticals which provide many alternatives to traditional medicine, and reduce the consensus of traditional knowledge for some common ailments. 
Table 1. Plant species used by Galo tribe for curing different ailments.

\begin{tabular}{|c|c|c|c|c|c|c|c|c|}
\hline Sl. No. & Illness category & Diseases & Scientific name & Family & Local name & $\begin{array}{l}\text { Part } \\
\text { used }\end{array}$ & Herbal formulation & Habit \\
\hline \multirow[t]{13}{*}{1.} & \multirow{13}{*}{$\begin{array}{l}\text { Dermatological } \\
\text { disorder }\end{array}$} & Inflammation & Angiopteris evecta (G. Forst.) Hoffm. & Marattiaceae & Tase & $\mathrm{Rh}$ & Juice and paste (E) & $\mathrm{F}$ \\
\hline & & \multirow{4}{*}{ Skin diseases } & Artocarpus heterophyllus Lamk. & Moraceae & Bella & $\mathrm{Lv}$ & Paste $(\mathrm{E})$ & $\mathrm{T}$ \\
\hline & & & Curcuma caesia Roxb. & Zingiberaceae & Kayane-take & $\mathrm{Rh}$ & Paste (E) & $\mathrm{H}$ \\
\hline & & & Drymaria cordata (L.) Willd. ex Schult. & Caryophyllaceae & Kiddi-kire & $\mathrm{Wp}$ & Paste (E) & $\mathrm{H}$ \\
\hline & & & Gynocardia odorata R. Br. & Flacourtiaceae & Takui & $\mathrm{Se}$ & $\begin{array}{l}\text { Extraction of seed oil } \\
\text { (E) }\end{array}$ & $\mathrm{T}$ \\
\hline & & \multirow{3}{*}{$\begin{array}{l}\text { Wound healing } \\
\text { and Cuts }\end{array}$} & Bambusa balcooa Roxb. & Poaceae & Hayi & Ogl & Juice and paste (E) & $\mathrm{S}$ \\
\hline & & & Mikania micrantha Kunth. & Asteraceae & $\begin{array}{l}\text { Saoun-sarman/ } \\
\text { Eyi namso }\end{array}$ & Lv & Past (E) & $\mathrm{C}$ \\
\hline & & & Ageratum conyzoides (L.) L. & Asteraceae & Rego & $\mathrm{Lv}$ & Juice and paste $(\mathrm{E})$ & $\mathrm{H}$ \\
\hline & & Scabies & Drymaria cordata (L.) Willd. ex Schult. & Caryophyllaceae & Kiddi-kire & $\mathrm{Wp}$ & Past (E) & $\mathrm{H}$ \\
\hline & & \multirow{3}{*}{$\begin{array}{l}\text { Abscesses and } \\
\text { allergy }\end{array}$} & Entada rheedii Spreng. & Fabaceae & Tase-polo & $\mathrm{Se}$ & Eaten raw (I) & $\mathrm{T}$ \\
\hline & & & Piper pedicellatum C.DC. & Piperaceae & Raro & $\mathrm{Lv}$ & $\begin{array}{l}\text { Decoction with water } \\
\text { (I) }\end{array}$ & $\mathrm{S}$ \\
\hline & & & Thunbergia coccinea Wall. & Acanthaceae & Pohak-rigo & Rt & Decoction (I) & $\mathrm{C}$ \\
\hline & & Relief burning & Stemona tuberosa Lour. & Stemonaceae & Pa-gore & $\mathrm{Rh}$ & Paste (E) & $\mathrm{C}$ \\
\hline \multirow[t]{17}{*}{2.} & \multirow{17}{*}{$\begin{array}{l}\text { Gastrointestinal } \\
\text { disorder }\end{array}$} & \multirow[t]{6}{*}{ Dysentery } & Artocarpus heterophyllus Lamk. & Moraceae & Bella & Lv & Decoction (I) & $\mathrm{T}$ \\
\hline & & & Citrus limon (L.) Osbeck & Rutaceae & Sipin & Fr & Juice (I) & $\mathrm{S}$ \\
\hline & & & Houttuynia cordata Thunb. & Saururaceae & Moyum-kneme & $\mathrm{Lv}$ & Decoction raw (I) & $\mathrm{H}$ \\
\hline & & & Melastoma malabathricum L. & Melastomaceae & Raja & Lv & Juice (I) & $\mathrm{S}$ \\
\hline & & & Musa balbisiana Colla. & Musaceae & Hulu & Fr & Raw (I) & $\mathrm{S}$ \\
\hline & & & Psidium guajava L. & Myrtaceae & Mudrandu & $\mathrm{Lv}$ & Raw (I) & $\mathrm{T}$ \\
\hline & & Constipation & Begonia roxburghii A.DC. & Begoniaceae & Buku-surbu & St & Decoction (I) & $\mathrm{H}$ \\
\hline & & \multirow[t]{4}{*}{ Diarrhea } & Citrus limon (L.) Osbeck. & Rutaceae & Sipin & $\mathrm{Fr}$ & Juice (I) & $\mathrm{S}$ \\
\hline & & & Houttuynia cordata Thunb. & Saururaceae & Moyum-kneme & Lv & Decoction, raw (I) & $\mathrm{H}$ \\
\hline & & & Melastoma malabathricum L. & Melastomaceae & Raja & Lv & Juice (I) & $\mathrm{S}$ \\
\hline & & & Musa balbisiana Colla. & Musaceae & Hulu & Fr & Raw (I) & $\mathrm{S}$ \\
\hline & & \multirow[t]{3}{*}{ Stomach pain } & Clerodendrum glandulosum Lindl. & Verbenaceae & Oing tapo & $\mathrm{Lv}$ & Decoction of leaves (I) & $\mathrm{S}$ \\
\hline & & & Curcuma caesia Roxb. & Zingiberaceae & Kayane-take & $\mathrm{Rh}$ & Juice (I) & $\mathrm{H}$ \\
\hline & & & Stemona tuberosa Lour. & Stemonaceae & Pa-gore & $\mathrm{Rh}$ & Paste and juice (I) & $\mathrm{C}$ \\
\hline & & \multirow{3}{*}{$\begin{array}{l}\text { Stomach } \\
\text { disorder }\end{array}$} & Elaeocarpus floribundus Blume & Elaeocarpaceae & Sur-sur & $\mathrm{Fr}$ & Eaten $\operatorname{raw}(\mathrm{I})$ & $\mathrm{T}$ \\
\hline & & & Clerodendrum glandulosum Lindl. & Verbenaceae & Oing tapo & $\mathrm{Lv}, \mathrm{Rt}$ & Decoction of leaves (I) & $\mathrm{S}$ \\
\hline & & & Curcuma caesia Roxb. & Zingiberaceae & Kayane-take & $\mathrm{Rh}$ & Juice (I), & $\mathrm{H}$ \\
\hline
\end{tabular}




\begin{tabular}{|c|c|c|c|c|c|c|c|c|}
\hline Sl. No. & Illness category & Diseases & Scientific name & Family & Local name & $\begin{array}{l}\text { Part } \\
\text { used }\end{array}$ & Herbal formulation & Habit \\
\hline & & \multirow{8}{*}{$\begin{array}{l}\text { Blood } \\
\text { dysentery }\end{array}$} & Eryngium foetidum $\mathrm{L}$. & Apiaceae & Rithak & Lv & Juice,boil (I) & $\mathrm{H}$ \\
\hline & & & Stemona tuberosa Lour. & Stemonaceae & Pa-gore & $\mathrm{Rh}$ & Paste and juice (I) & $\mathrm{C}$ \\
\hline & & & Garcinia lanceifolia Roxb. & Clusiaceae & Takter & Fr & Decoction with water (I) & $\mathrm{T}$ \\
\hline & & & Litsea cubeba (Lour) Pers. & Lauraceae & Tayer & $\mathrm{Se}$ & Decoction (I) & $\mathrm{T}$ \\
\hline & & & Musa velutina $\mathrm{H}$.Wendl. and Drude. & Musaceae & Kodak & $\operatorname{Inf}$ & $\operatorname{Raw}(\mathrm{I})$ & $\mathrm{S}$ \\
\hline & & & Curcuma caesia Roxb. & Zingiberaceae & Kayane-take & $\mathrm{Rh}$ & Juice(I) & $\mathrm{H}$ \\
\hline & & & Garcinia pedunculata Roxb. Ex Wight. & Clusiaceae & Liba & $\mathrm{Fr}$ & Decoction with water(I) & $\mathrm{T}$ \\
\hline & & & Musa $\times$ paradisiaca $\mathrm{L}$ & Musaceae & Hulu & $\mathrm{Fr}$ & Raw (I) & $\mathrm{S}$ \\
\hline & & \multirow[t]{3}{*}{ Gastric } & Carica papaya $\mathrm{L}$. & Caricaceae & Papaya & $\mathrm{La}$ & Juice (I) & $\mathrm{T}$ \\
\hline & & & Mentha spicata L. & Lamiaceae & Podina & $\mathrm{Lv}$ & Juice (I) & $\mathrm{H}$ \\
\hline & & & Stemona tuberosa Lour. & Stemonaceae & Pa-gore & $\mathrm{Rh}$ & Paste and juice (I) & $\mathrm{C}$ \\
\hline & & Acidity & Mentha spicata L. & Lamiaceae & Podina & Le & Juice (I) & $\mathrm{H}$ \\
\hline & & Intestinal worm & Capsicum chinense Jacq. & Solanaceae & Mane yaluk & $\mathrm{Fr}$ & Eaten raw (I) & $\mathrm{H}$ \\
\hline & & & $\begin{array}{l}\text { Acmella paniculata (Wall. ex DC.) } \\
\text { R.K.Jansen }\end{array}$ & Asteraceae & Marsa & $\begin{array}{l}\text { Ys } \\
\text {,Fl }\end{array}$ & Direct chewed (I) & $\mathrm{H}$ \\
\hline & & & Lasia spinosa (L.) Thwaites & Araceae & Rubi & St & Decoction (I) & $\mathrm{H}$ \\
\hline & & & Gynocardia odorata R. Br. & Flacourtiaceae & Takui & $\mathrm{Se}$ & Extraction of seed oil (E) & $\mathrm{T}$ \\
\hline \multirow[t]{4}{*}{3.} & \multirow{4}{*}{$\begin{array}{l}\text { Respiratory } \\
\text { system disorder }\end{array}$} & Cough & Artemisia indica Willd. & Asteraceae & Tapen & $\mathrm{Lv}$ & Juice (I) & $\mathrm{H}$ \\
\hline & & & Litsea cubeba (Lour.) Pers. & Lauraceae & Tayer & $\mathrm{Se}$ & Decoction (I) & $\mathrm{T}$ \\
\hline & & & Piper mullesua Buch.-Ham. ex D. Don & Piperaceae & Pinee & $\mathrm{Fr}$ & Direct chewed (I) & $\mathrm{S}$ \\
\hline & & Asthma & Trichosanthes tricuspidata Lour. & Cucurbitaceae & Sojar-Momar & $\mathrm{Fr}$ & Juice (I) & $\mathrm{C}$ \\
\hline \multirow[t]{8}{*}{4.} & \multirow{8}{*}{$\begin{array}{l}\text { Genitourinary } \\
\text { system disorder }\end{array}$} & Easy delivery & Carica papaya L. & Caricaceae & Papaya & $\mathrm{La}$ & Juice (I) & $\mathrm{T}$ \\
\hline & & Abortion & Meyna laxiflora Robyns & Rubiaceae & Kutka & Fr & Raw (I) & $S$ \\
\hline & & Leucorrhoea & $\begin{array}{l}\text { Acmella paniculata (Wall. ex DC.) } \\
\text { R.K.Jansen }\end{array}$ & Asteraceae & Marsa & $\begin{array}{l}\text { Ys } \\
\text {,Fl }\end{array}$ & Direct chewed (I) & $\mathrm{H}$ \\
\hline & & Painful & Tamarindus indica $\mathrm{L}$. & Caesalpinaceae & Imli & Lv & Juice (I) & $\mathrm{T}$ \\
\hline & & menstruation & Artemisia indica Willd. & Asteraceae & Tapen & $\mathrm{Lv}$ & Juice (I) & $\mathrm{H}$ \\
\hline & & Pregnancy & Colocasia esculenta (L.)Schott. & Araceae & Rubita & Sh & Decoction (I) & $\mathrm{H}$ \\
\hline & & Urinary problems & Paederia foetida $\mathrm{L}$. & Rubiaceae & Aape -tare & Lv & Raw (I) & $\mathrm{C}$ \\
\hline & & & Persicaria chinensis (L.) H. Gross & Polygonaceae & Diko-tamu & Lv & Boil (I) & $\mathrm{H}$ \\
\hline \multirow[t]{3}{*}{5.} & \multirow[t]{3}{*}{ Fever } & Fever & Diplazium esculentum (Retz) Sw. & Athyriaceae & Takka-paya & $\mathrm{Rh}$ & Decoction (I) & $\mathrm{H}$ \\
\hline & & & Garcinia lanceifolia Roxb. & Clusiaceae & Takter & Fr & Decoction with water(I) & $\mathrm{T}$ \\
\hline & & & Mikania micrantha Kunth. & Asteraceae & $\begin{array}{l}\text { Saoun-sarman } \\
\text { / Eyi namso }\end{array}$ & Lv & Past (E) & $\mathrm{C}$ \\
\hline
\end{tabular}




\begin{tabular}{|c|c|c|c|c|c|c|c|c|}
\hline Sl. No. & Illness category & Diseases & Scientific name & Family & Local name & $\begin{array}{l}\text { Part } \\
\text { used }\end{array}$ & Herbal formulation & Habit \\
\hline \multirow[t]{7}{*}{6.} & \multirow[t]{7}{*}{ Pain } & \multirow[t]{2}{*}{ Body pain } & Cyclosorus parasiticus (L.) Farwell. & Thelypteridaceae & Ruk-dik & Lv & Paste (E) & $\mathrm{H}$ \\
\hline & & & Artemisia indica Willd. & Asteraceae & Tapen & Lv & Juice(I) & $\mathrm{H}$ \\
\hline & & \multirow[t]{2}{*}{ Headache } & Houttuynia cordata Thunb. & Saururaceae & $\begin{array}{l}\text { Moyum- } \\
\text { kneme }\end{array}$ & Lv & Decoction $\operatorname{raw}(\mathrm{I})$, & $\mathrm{H}$ \\
\hline & & & Clerodendrum glandulosum Lindl. & Verbenaceae & Oing tapo & $\mathrm{Lv}, \mathrm{Rt}$ & Leaf and root paste $(\mathrm{E})$ & $\mathrm{S}$ \\
\hline & & \multirow[t]{3}{*}{ Toothache } & Zanthoxylum armatum DC. & Rutaceae & Rikon & St & Direct chewed (I) & $\mathrm{T}$ \\
\hline & & & Solanum aculeatissimum Jacq. & Solanaceae & Sot-bayom & $\mathrm{Fr}$ & $\begin{array}{l}\text { Direct chewed with } \\
\text { chili (I) }\end{array}$ & $\mathrm{S}$ \\
\hline & & & Paederia foetida $\mathrm{L}$. & Rubiaceae & Aape -tare & $\mathrm{Lv}$ & $\operatorname{Raw}(\mathrm{I})$ & $\mathrm{C}$ \\
\hline \multirow[t]{2}{*}{7.} & \multirow[t]{2}{*}{ Jaundice } & \multirow[t]{2}{*}{ Jaundice } & Houttuynia cordata Thunb. & Saururaceae & $\begin{array}{l}\text { Moyum- } \\
\text { kneme }\end{array}$ & Lv & Decoction raw (I) & $\mathrm{H}$ \\
\hline & & & Drymaria cordata (L.) Willd. ex Schult. & Caryophyllaceae & Kiddi-kire & $\mathrm{Wp}$ & Past (E) & $\mathrm{H}$ \\
\hline \multirow[t]{11}{*}{8.} & \multirow[t]{11}{*}{ General health } & Antidotes & Paederia foetida $\mathrm{L}$ & Rubiaceae & Aape -tare & $\mathrm{Lv}$ & Raw (I) & $\mathrm{C}$ \\
\hline & & $\begin{array}{l}\text { High blood } \\
\text { pressure }\end{array}$ & Clerodendrum glandulosum Lindl. & Verbenaceae & Oing tapo & $\mathrm{Lv}$ & Decoction (I) & $\mathrm{S}$ \\
\hline & & Nose bleeding & Artemisia indica Willd. & Asteraceae & Tapen & Lv & Juice (I) & $\mathrm{H}$ \\
\hline & & \multirow[t]{2}{*}{ Pneumonia } & Entada rheedii Spreng. & Fabaceae & Tase-polo & $\mathrm{Se}$ & Eaten raw (I) & $\mathrm{T}$ \\
\hline & & & Trichosanthes tricuspidata Lour. & Cucurbitaceae & Sojar-Momar & $\mathrm{Fr}$ & Juice (I) & $\mathrm{C}$ \\
\hline & & \multirow[t]{2}{*}{ Tonsillitis } & Houttuynia cordata Thunb. & Saururaceae & $\begin{array}{l}\text { Moyum- } \\
\text { kneme }\end{array}$ & Lv & Decoction raw (I) & $\mathrm{H}$ \\
\hline & & & Thunbergia coccinia Wall. & Acanthaceae & Pohak-rigo & $\mathrm{Rt}$ & Decoction (I) & $\mathrm{C}$ \\
\hline & & \multirow[t]{2}{*}{ Mouth ulcer } & Piper mullesua Buch.-Ham. ex D. Don & Piperaceae & Pinee & $\mathrm{Fr}$ & Direct chewed (I) & $\mathrm{S}$ \\
\hline & & & $\begin{array}{l}\text { Acmella paniculata (Wall. ex DC.) } \\
\text { R.K.Jansen }\end{array}$ & Asteraceae & Marsa & $\begin{array}{l}\text { Ys } \\
\text {,Fl }\end{array}$ & Direct chewed (I) & $\mathrm{H}$ \\
\hline & & Hair fall & Dillenia indica $\mathrm{L}$ & Dilleniaceae & Sampak & Fr & Raw $(E)$ & $\mathrm{T}$ \\
\hline & & $\begin{array}{l}\text { Dumbness in young } \\
\text { children }\end{array}$ & Asplenium nidus L. & Aspleniaceae & Gabo-lagboi & Lv & Decoction (I) & $\mathrm{F}$ \\
\hline
\end{tabular}

\section{Abbreviation:}

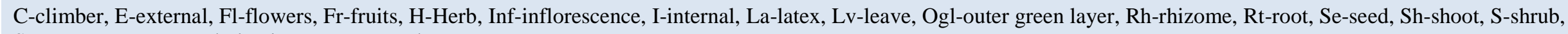
St-stem, T-tree, Wp-whole plant, Ys-young shoots. 


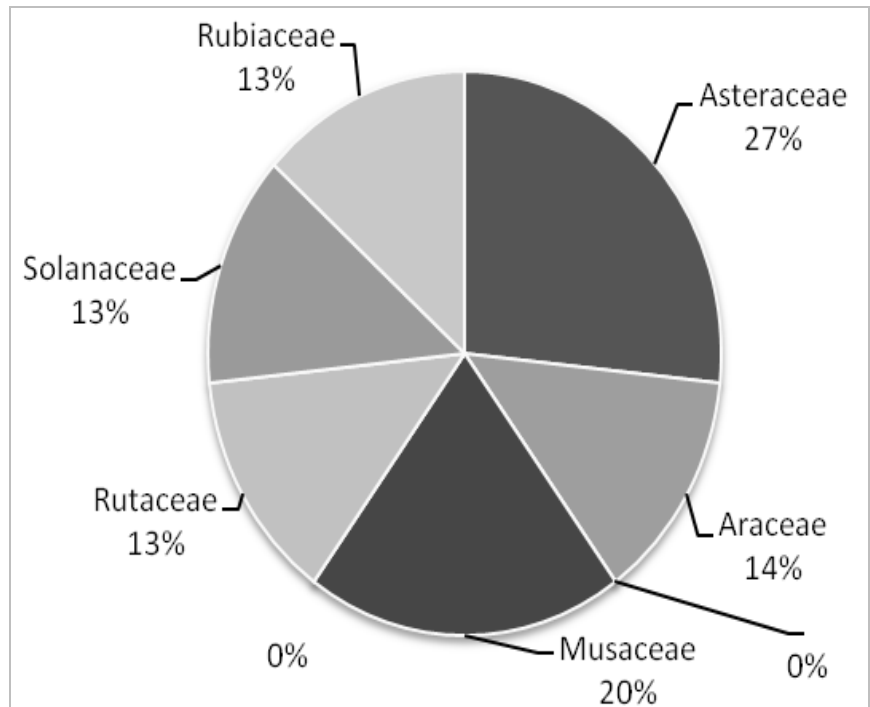

Fig. 2: Pie diagram showing percentage distribution of six major dominant families.

The low consensus (Fic0.57) in genito-urinary system disorder may be lackof knowledgeof this disease among male informers. Although the majority of the species reported as ethno medicinal plants used by Galo are also known to be used by other ethnic groups of the region. Among the ethno medicinal plants 30 plant species including Ageratum conyzoides, Bambusa balcooa, Clerodendron colebrookianum, Colocasia esculenta, Eryngium foetidum, Garcinia pedunculata, Houttouynia cordata,Mikania micrantha, Paederia foetida, Spilanthes paniculata etc. are usedfor common diseases likeblood clotting, wound healing, dysentery, cut, gastric, cough, are also found to be similar used by other communities of the state as reported by various authors (Mutem and Das, 2005; Srivastava and Adi Community, 2009; Das and Tag, 2006; Bhuyan, 2007; Panda and Srivastava, 2010).

Although a large number of plant species are used against various ailments by the community but the utility pattern or specific uses of many species reported in the present study were not known earlier and hence it forms the first report of the species for the use recorded in the present study. The uses of Trichosanthes tricuspidata for asthma and pneumonia Clerodendron colebrookianumis for headache Carica papaya for gastric ulcer, Bambusa balcooa for wound healing, Capsicum chinense for intestinal worm, Citrus limon for diarrhea, dysentery and vomiting tendency, Garcinia lancifolia for Stomach disorder and fever; Musa bulbisiana for dysentery and diarrhea, Meyna laxiflora for Abortion, Piper pedicellatum for skin abscesses, Polygonum chinenseis for urine problem, Stemona tuberose for gastric and stomach disorder, relief burning, Thunbergia coccinia for tonsil and skin abscesses are some of them.

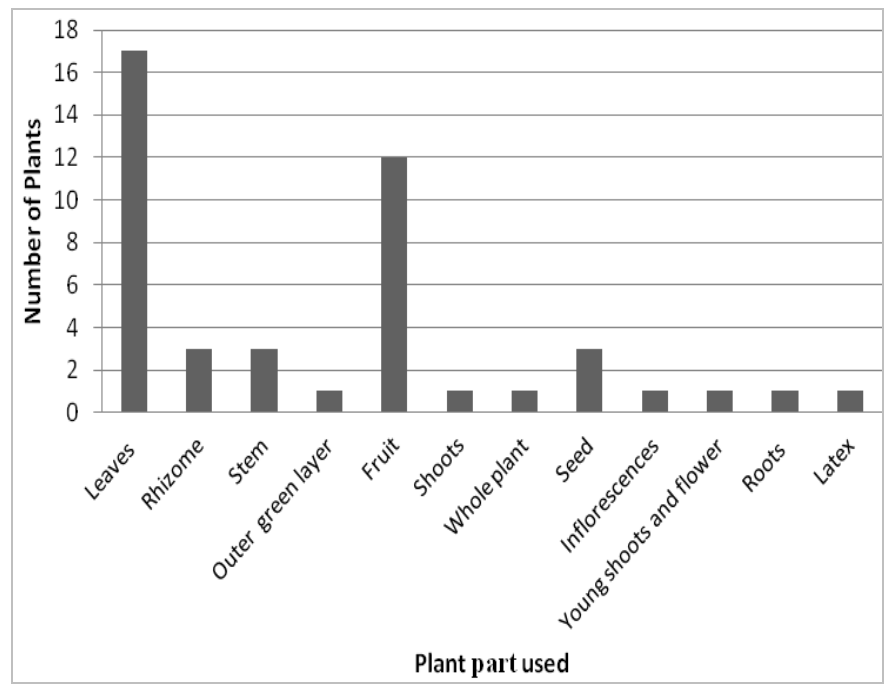

Fig. 3: Histogram showing plant parts used as medicine.

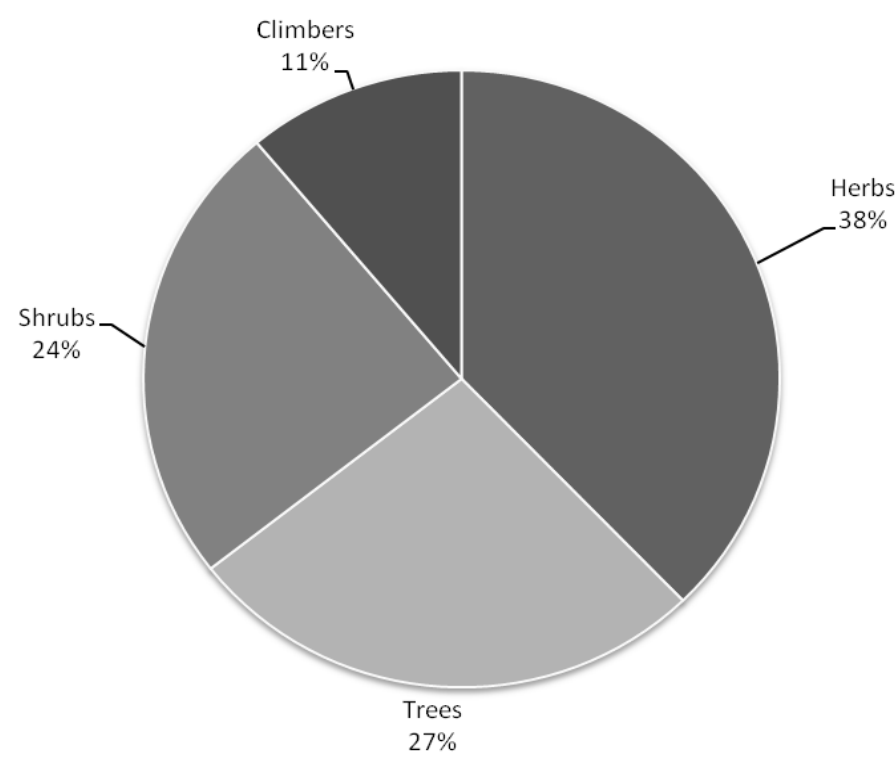

Fig. 4: Pie diagram showing habit wise distribution of medicinal plants.

In the present study, general health category is included as theyuse plants in their diets to maintain good health. The general health category shows relatively high level of consensus (Table 1). From the earlier time Galos have tradition to eat certain plants as vegetable (Spilanthes paniculata, Clerodendron colebrookianum, Paederia foetida, Dillenia indica, etc.) and some others are as spices and condiments (Houttouynia cordata, Menthe spicata, Zanthoxylum armatum, Paederia foetida, Eryngium foetidum, etc.) on a regular basis according to variation in season in order to prevent certain diseases. 

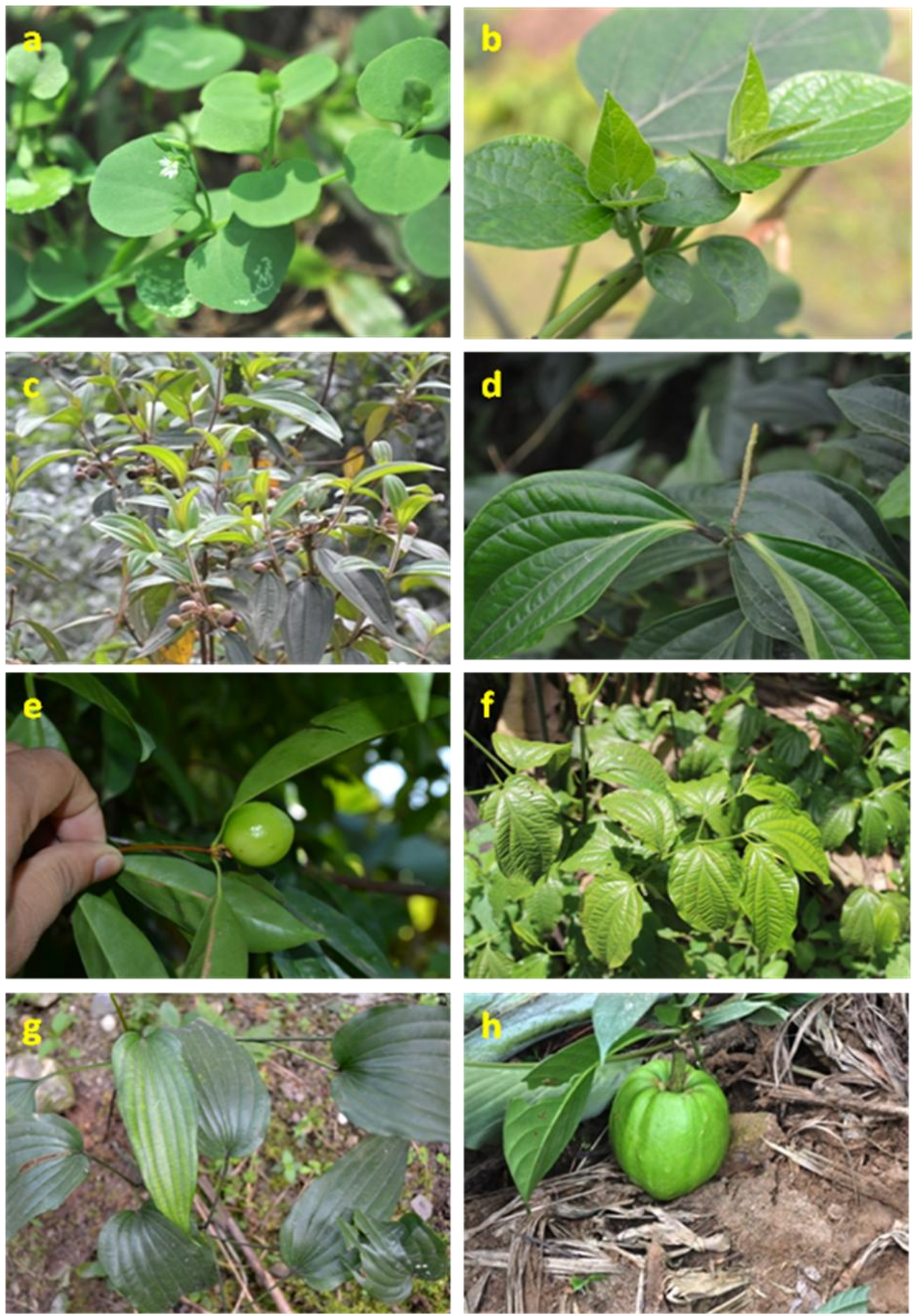

Fig. 5: Medicinal plants used by Galo tribe (a-h): Drymaria cordata (a); Clerodendrum glandulosum (b); Melastoma malabathricum (c); Piper mullesua (d); Garcinia lanceifolia (e); Piper pedicellatum (f); Stemona tuberose (g); Garcinia pedunculata (h). 
Table 2. Informant consensus of medicinal plants of different ailment categories.

\begin{tabular}{|c|c|c|c|c|}
\hline $\begin{array}{l}\text { Sl. } \\
\text { No. }\end{array}$ & Illness category (Diseases and disorders) & $\begin{array}{l}\text { Number of } \\
\text { taxa } \\
\left(\mathbf{N}_{t}\right)\end{array}$ & $\begin{array}{l}\text { Number of use } \\
\text { reports } \\
\left(\mathbf{N}_{u r}\right)\end{array}$ & $\begin{array}{l}\text { Informant's } \\
\text { consensus index } \\
\text { factor }\left(\mathbf{F}_{\mathrm{IC}}\right)\end{array}$ \\
\hline 1 & $\begin{array}{l}\text { Dermatological disorder (Inflammation, skin diseases, wound } \\
\text { healing, cuts, scabies, Abscesses and allergy, relief burning,) }\end{array}$ & 12 & 65 & 0.82 \\
\hline 2 & $\begin{array}{l}\text { Gastrointestinal disorder (Dysentery, constipation, diarrhea, } \\
\text { stomach disorder, blood dysentery, gastric, acidity, intestinal } \\
\text { worms. }\end{array}$ & 25 & 97 & 0.75 \\
\hline 3 & Respiratory system disorder (cough, asthma ) & 4 & 10 & 0.66 \\
\hline 4 & $\begin{array}{l}\text { Genitourinary system disorder (easy delivery, abortion, } \\
\text { leucorrhoea, painful menstruation, pregnancy, urinary problems) }\end{array}$ & 7 & 15 & 0.57 \\
\hline 5 & Fever (Fever ) & 3 & 5 & 0.5 \\
\hline 6 & Pain (body pain, headache, toothache) & 7 & 22 & 0.71 \\
\hline 7 & Jaundice (jaundice) & 2 & 4 & 0.66 \\
\hline 8 & $\begin{array}{l}\text { General health (Antidotes, high blood pressure, nose bleeding, } \\
\text { pneumonia, tonsillitis, mouth ulcer, hair fall, Dumbness in } \\
\text { young children.) }\end{array}$ & 12 & 55 & 0.79 \\
\hline
\end{tabular}

\section{Conclusion}

The present study reveals the use of medicinal plant in traditional medicine has a significant role in providing the primary healthcare needs of the Galo ethnic group of West Siang district. Remoteness of the place acceptance of traditional medicine and in accessibility to modern healthcare facilities could be considered as the main factors for the continuation of the practice. 45 number of plant species belonging to 32 families and 41 genera were documented against 8different categories of ailments. The highest number of plant species was reported to be used for treatment of Dysentery. This study contributes to the enormous indigenous knowledge on medicinal plants and plant-based remedies practiced among ethnic groups. There is a lack of written documentation of traditional healing knowledge and transmission of the knowledge to the future generation takes place only through oral communication. Also a lack of interest is observed among younger generation to pursue to the practice of traditional healing. Therefore, there is an immediate need for conservation of the ethnomedicinal knowledge of tribal people. The Government, NGO's and other organization should take part in the process of conservation through awareness and training programs.

\section{Conflict of interest statement}

Authors declare that they have no conflict of interest.

\section{Acknowledgement}

Authors are thankful to NEC, Govt. of India, Shillong for financial assistance and to all Galo informants for sharing their valuable information. We are also thankful to the Director, NERIST for laboratory and research facilities.

\section{References}

Arwa, S.P., Nyunja, R.O., Onyango, J.C. 2010. Plant Species in the Folk medicine of Kit Mikayi Region, Western Kenya. Ethnobot. Leaflets. 14, 836-40.

Bhuyan, L.R., 2007. Some ethnomedicinal plants of Arunachal Pradesh. Bull. Arun. Fo. Res. 23(1and2), 45-50.

Bora, S.S., Lahan, J.P., Barooah, M., Sarmah, R., 2012. Poka-a traditional rice wine of the Galo tribe of Arunachal Pradesh, India. Int. J. Agri. Sci. 4(6), 268-271.

Choudhery, H.J., 1996. Materials for the flora of Arunachal Pradesh. Kolkata: Botanical Survey of India.

Dagyom, K., Gopi, G.V., 2006. Ethnozoology of Galo tribe with special reference to edible insects in Arunachal Pradesh. Ind. J. Trad. Know. 8 (1), 81-83.

Das, A. K., Tag, H., 2oo6. Ethnomedicinal studies of the Khamti tribe of Arunachal Pradesh. Ind. J. Trad. Know. 5(3), 317-322.

Das, M., Jaishi, A., Sarma, H.N.,2013.Traditional medicines of herbal origin practice by the Adi tribe of East Siang District of Arunachal Pradesh, Ind. Glob. J. Res. Med. Plants Ind. Med. 2 (5), 298-310.

Doley, B., Gajurel, P.R., Rethy, P., Singh, B. Buragohain, R., Potsangbam, S., 2010. Less known ethno medicinal plants used by the Nyishi community of Papum Pare District, Arunachal Pradesh. J. Bio. Sci. Res. 1, 34-36.

Dutta, R., Bhattacharjya, K.B., 2005. An indigenous community fishing practice of Tirap district Arunachal Pradesh. Ind. J. Trad. Know. 7,624-626.

Goswami, P., Soki, D., Jaishi, A., Das, M., Sarma, H. N.2009. Traditional healthcare practices among the Tagin tribe of Arunachal Pradesh, Ind. J. Trad. Know. 8,127-130. 
Heinrich, M., 2000. Ethnobotany and its role in drug development. Phytother Res. 14,479-488.

Heinrich, M., Ankli, A., Frei, B., Weimann, C., Sticher O., 1998. Medicinal plants in Mexico: healers' consensus And cultural importance. Soc. Sci. Med. 47(11), 1859-1871.

Hooker, J.D., 1894. Flora of British India, Vol 6. Ashford, Kent, England: L Reeve and Co.Ltd.,NR.

Jeri, L., Tag, H., Tsering, J., Katila, P., Mingki, T., Das, A.K., 2011. Ethnobotanical Investiagation of Edible and Medicinal Plants in Pakke Wildlife Sanctuary of East Kameng District in Arunachal Pradesh, India. Pleione, $5(1), 83-90$.

Kala, C.P.2005. Ethnomedicinal botany of the Apatani in the Eastern Himalayan region of India, J. Ethnobio. Ethnomed.1,11.

Khongsai, M., Saikia, S.P., Kayang, H., 2011. Ethnomedicinal plants used by different tribes of Arunachal Pradesh. Ind. J. Trad. Know. 10 (3), 541-546.

Kohli, Y.P.,2001. Non traditional foods and ethnobotanical plants of Lower Subansiri district, Arunachal Pradesh. Arun. Fo. News. 19(1and2), 169-171.

Mutem, G., Das, A. K., 2005. Traditional medicinal plants of Nyishi tribe of Arunachal Pradesh. Aru. Fo. News. 21(1and2),31-43.

Namsa, N. D., Mandal, M., Tangjang, S., Mandal S.C., 2011. Ethnobotany of the Monpa ethnic group at Arunachal Pradesh, India. J, Ethnobio. Ethnomed. 7,31.

Nimachow, G., Rawat, J.S., Arunachalam, A., Dai, O., 2011. Ethno-medicines of Aka tribe, West Kameng District, Arunachal Pradesh (India). Sci. Cult. 77(3-4), 149-155.

Nimasow, G., Ngupok, R., Nimasow, O.D., 2012. Ethnomedicinal knowledge among the Adi tribes of lower Dibang valley district of Arunachal Pradesh, India. Int. Res. J. Pharm. 3(6).
Njoroge, G.N., Bussmann, R. W., 2006. Herbal usage and informant consensus in ethnoveterinary management cattle diseases among the Kikuyus (Central Kenya), J. Ethnopharm. 108(3), 332-9.

Panda, S., Srivastava, R. C., 2010. New ethnomedicinal practices by the Akas,Nepalese and Dirang Monpas of West Kameng District in Arunachal Pradesh. Ind. J. Trad. Know. 9(4), 721-723.

Post, M. W., 2007. A Grammar of Galo. Ph. D.thesis, La Trobe University, Bundoora, Victoria 3086, Australia.

Sen, P., Dollo, M., Choudhury, M. D., Choudhury, D.2009. Documentation of traditional herbal knowledge of Khamptis of Arunachal Pradesh. Ind. J. Trad. Know.7, 438-442.

Srivastava, R. C., Adi Community, 2009. Traditional knowledge of Adi tribe of Arunachal Pradesh on plants, Ind. J. Trad. Knowl. 8(2), 146-153.

Srivastava, R. C., Nyshi Community, 2010. Traditional knowledge of Nyshi (Daffla) tribe of Arunachal Pradesh. Ind. J. Trad. Knowl. 9 (1), 26-37.

Tag, H., Das, A. K., 2004.Ethnobotanical notes on Hill Miri Tribe of Arunachal Pradesh. Ind. J. Trad. Know. 3, 80-85.

Tarak, D., Koyu, R., Samal P.K., Singh, S.P., 2009. Wild vegetable plants used by galo tribe of West Siang district, Arunachal Pradesh (India), Bull. Arun. Fo. Res. 25 (1 and2), 34-36.

Tolossa, K., Debela, E., Athanasiadou, S., Tolera, A., Ganga, G., Houdijk, J.G. M., 2013. Ethno-medicinal study of plants used for treatment of human and livestock ailments by traditional healers in South Omo, Southern Ethiopia. J. Ethnobiol. Ethnomed. 9, 32.

\section{How to cite this article:}

Bharali, P., Singh, B., Sharma, C. L., 2016. Ethnomedicinal knowledge of Galo tribe from Arunachal Pradesh, India. Int. J. Curr. Res. Biosci. Plant Biol. 3(6), 139-148. doi: http://dx.doi.org/10.20546/ijcrbp.2016.306.017 